\title{
POLYNOMIALS AND NUMERICAL RANGES
}

\author{
CHI-KWONG LI
}

(Communicated by Louis J. Ratliff, Jr.)

\begin{abstract}
Let $A$ be an $n \times n$ complex matrix. For $1 \leq k \leq n$ we study the inclusion relation for the following polynomial sets related to the matrix $A$.

(a) The classical numerical range of the $k$ th compound of the matrix $\lambda I-A$.

(b) The $k$ th decomposable numerical range of the matrix $\lambda I-A$.

(c) The convex hull of the set of all monic polynomials of degree $k$ that divide the characteristic polynomial of $A$.

Moreover, we give an example showing that the set described in (a) is not convex in general. This settles a question raised by C. Johnson.
\end{abstract}

Results and examples. Let $\mathbf{C}_{n \times n}$ be the set of all $n \times n$ complex matrices. For $A \in \mathbf{C}_{n \times n}$, its (classical) numerical range is the set

$$
W(A)=\left\{x A x^{*}: x \in \mathbf{C}^{n} \text { and } x x^{*}=1\right\} .
$$

Suppose $A$ is hermitian and its characteristic polynomial $p_{A}(\lambda)=\operatorname{det}(\lambda I-A)$ has roots $\lambda_{1} \geq \cdots \geq \lambda_{n}$. For $1 \leq k \leq n$, we denote by $\operatorname{Int}\left(p_{A}(\lambda) ; k\right)$ the set of all monic polynomials $q(\lambda)$ of degree $k$ that have $k$ real roots $\mu_{1} \geq \cdots \geq \mu_{k}$ satisfying

$$
\lambda_{i} \geq \mu_{i} \quad \text { and } \quad \mu_{k-i+1} \geq \lambda_{n-i+1} \quad \text { for } i=1, \ldots, k \text {. }
$$

Let $P_{k}(\lambda I-A)$ be the convex hull of the set

$$
\left\{\prod_{j=1}^{k}\left(\lambda-\lambda_{i_{j}}\right): 1 \leq i_{1}<\cdots<i_{k} \leq n\right\} .
$$

Johnson [3] proved the interesting result that

$$
\operatorname{Int}\left(p_{A}(\lambda) ; n-1\right)=W(\operatorname{adj}(\lambda I-A))=P_{n-1}(\lambda I-A) .
$$

For $B \in \mathbf{C}_{n \times n}$ and $1 \leq k \leq n$, let $C_{k}(B)$ be the $k$ th compound of $B$ (see [4] for definitions and properties). Since $W\left(B^{t}\right)=W(B)$ and $C_{n-1}(B)$ is unitarily similar to $\operatorname{adj}(B)^{t}$, we may replace " $W(\operatorname{adj}(\lambda I-A))$ " by " $W\left(C_{n-1}(\lambda I-A)\right)$ " in (1). Clearly,

$$
\begin{aligned}
\operatorname{Int}\left(p_{A}(\lambda) ; 1\right) & =\left\{\lambda-\mu: \lambda_{1} \geq \mu \geq \lambda_{n}\right\} \\
& =\{\lambda-\mu: \mu \in W(A)\} \\
& =W(\lambda I-A)=W\left(C_{1}(\lambda I-A)\right),
\end{aligned}
$$

Received by the editors August 10, 1987 and, in revised form, November 20, 1987.

1980 Mathematics Subject Classification (1985 Revision). Primary 15A60, 15A69, 26 C10.

Key words and phrases. Numerical range, decomposable numerical range, characteristic polynomial.

Research supported by NSF grant DMS-8521521. 
and

We have

$$
\operatorname{Int}\left(p_{A}(\lambda) ; k\right)=W\left(C_{k}(\lambda I-A)\right)=P_{k}(\lambda I-A)
$$

for $k=1, n-1, n$.

Related to $W\left(C_{k}(B)\right)$ is the concept of the $k$ th decomposable numerical range of the matrix $B$ defined by (see [5])

$$
W_{k}^{\wedge}(B)=\left\{x C_{k}(B) x^{*}: x \in \bigwedge^{k} \mathbf{C}^{n} \text { is decomposable and } x x^{*}=1\right\},
$$

where $\bigwedge^{k} \mathbf{C}^{n}$ is the $k$ th Grassman space over $\mathbf{C}^{n}$. Evidently,

$$
W_{k}^{\wedge}(B) \subseteq W\left(C_{k}(B)\right) \text {. }
$$

It is known (see [4]) that equality in (3) holds for $k=1, n-1, n$. So (2) may be rewritten as

$$
\operatorname{Int}\left(p_{A}(\lambda) ; k\right)=W_{k}^{\wedge}(\lambda I-A)=W\left(C_{k}(\lambda I-A)\right)=P_{k}(\lambda I-A)
$$

for $k=1, n-1, n$.

For general $k$ we have the following theorem.

THEOREM 1. Let $A \in \mathbf{C}_{n \times n}$ be hermitian. Then for $1 \leq k \leq n$,

$$
\operatorname{Int}\left(p_{A}(\lambda) ; k\right)=W_{k}^{\wedge}(\lambda I-A) \subseteq W\left(C_{k}(\lambda I-A)\right)=P_{k}(\lambda I-A) .
$$

All four sets are equal if and only if the set

$$
\operatorname{Int}\left(p_{A}(\lambda) ; k\right)=W_{k}^{\wedge}(\lambda I-A)
$$

is convex.

ProOF. For $B \in \mathbf{C}_{n \times n}$, let $B[k]$ denote the $k \times k$ leading principal submatrix of $B$. Then (see [5]),

$$
W_{k}^{\wedge}(\lambda I-A)=\left\{\operatorname{det}\left(\left(\lambda I-U A U^{*}\right)[k]\right): U \text { unitary }\right\} .
$$

It follows that $q(\lambda) \in W_{k}^{\wedge}(\lambda I-A)$ if and only if $q(\lambda)$ is the characteristic polynomial of a matrix of the form $\left(U A U^{*}\right)[k]$, where $U$ is a unitary matrix. By a result of Fan and Pall [2],

The inclusion

$$
\operatorname{Int}\left(p_{A}(\lambda) ; k\right)=W_{k}^{\wedge}(\lambda I-A)
$$

$$
W_{k}^{\wedge}(\lambda I-A) \subseteq W\left(C_{k}(\lambda I-A)\right)
$$

is clear. Now consider $W\left(C_{k}(\lambda I-A)\right)$. Since

$$
W\left(C_{k}(\lambda I-A)\right)=W\left(C_{k}\left(U(\lambda I-A) U^{*}\right)\right)
$$

for any unitary matrix $U$, we may assume that

$$
\lambda I-A=\operatorname{diag}\left(\lambda-\lambda_{1}, \ldots, \lambda-\lambda_{n}\right) .
$$

It follows that $C_{k}(\lambda I-A)$ is a diagonal matrix with diagonal entries $\prod_{j=1}^{k}\left(\lambda-\lambda_{i_{j}}\right)$ with $1 \leq i_{1}<\cdots<i_{k} \leq n$. One easily checks that the numerical range of a diagonal matrix equals the convex hull of the diagonal entries. So

$$
W\left(C_{k}(\lambda I-A)\right)=P_{k}(\lambda I-A) \text {. }
$$


Finally, if the four sets are equal, then clearly the set $\operatorname{Int}\left(p_{A}(\lambda) ; k\right)=W_{k}^{\wedge}(\lambda I-A)$ is convex. Conversely, suppose $\operatorname{Int}\left(p_{A}(\lambda) ; k\right)=W_{k}^{\wedge}(\lambda I-A)$ is convex. By the fact that

$$
\prod_{j=1}^{k}\left(\lambda-\lambda_{i_{j}}\right) \in \operatorname{Int}\left(p_{A}(\lambda) ; k\right) \quad \text { for } 1 \leq i_{1}<\cdots<i_{k} \leq n
$$

we have

$$
P_{k}(\lambda I-A) \subseteq \operatorname{Int}\left(p_{A}(\lambda) ; k\right),
$$

and hence the four sets are equal.

We show that the four sets are not equal in general by the following example.

EXAMPLE 1. Let $A=\operatorname{diag}(1,2,3,4)$. Then

$$
p(\lambda)=(\lambda-1)(\lambda-2), \quad q(\lambda)=(\lambda-3)(\lambda-4) \in \operatorname{Int}\left(p_{A}(\lambda) ; 2\right) .
$$

However, the polynomial $(p(\lambda)+q(\lambda)) / 2$ has no real roots and thus does not belong to $\operatorname{Int}\left(p_{A}(\lambda) ; 2\right)$. So

$$
\operatorname{Int}\left(p_{A}(\lambda) ; 2\right) \neq P_{2}(\lambda I-A) .
$$

As mentioned in the proof of Theorem $1, W_{k}^{\wedge}(\lambda I-A)$ is the set of all characteristic polynomials of matrices in the form $\left(U A U^{*}\right)[k]$, where $U$ is unitary. For a hermitian matrix $A, W_{k}^{\wedge}(\lambda I-A)$ is simply $\operatorname{Int}\left(p_{A}(\lambda) ; k\right)$. For general matrices $A$, we do not have such a nice equivalence form for $W_{k}^{\wedge}(\lambda I-A)$. Anyway, one may still consider the sets $W\left(C_{k}(\lambda I-A)\right)$ and $P_{k}(\lambda I-A)$. In particular, we may extend Theorem 1 to

THEOREM 2. Let $A \in \mathbf{C}_{n \times n}$ be a normal matrix. Then

$$
W_{k}^{\wedge}(\lambda I-A) \subseteq W\left(C_{k}(\lambda I-A)\right)=P_{k}(\lambda I-A) .
$$

The three sets are equal if and only if the set $W_{k}^{\wedge}(\lambda I-A)$ is convex.

Proof. Similar to that of Theorem 1.

For nonnormal matrices $A$, we have no inclusions between the sets $W\left(C_{k}(\lambda I-A)\right)$ and $P_{k}(\lambda I-A)$. Moreover, the set $W\left(C_{k}(\lambda I-A)\right)$ may fail to be convex as shown in the following examples.

EXAMPLE 2. Let

$$
A=\left[\begin{array}{ll}
0 & 2 \\
0 & 0
\end{array}\right]
$$

Then

$$
P_{1}(\lambda I-A)=\{\lambda\} \varsubsetneqq\{\lambda-\mu:|\mu| \leq 1\}=W\left(C_{1}(\lambda I-A)\right) .
$$

EXAMPLE 3. Let

$$
A=\left[\begin{array}{cccc}
0 & -1 & 0 & 0 \\
0 & 0 & -1 & 1 \\
0 & 0 & 0 & -1 \\
0 & 0 & 0 & 0
\end{array}\right]
$$

Then

$$
\lambda I-A=\left[\begin{array}{cccc}
\lambda & 1 & 0 & 0 \\
0 & \lambda & 1 & -1 \\
0 & 0 & \lambda & 1 \\
0 & 0 & 0 & \lambda
\end{array}\right]
$$


and

$$
C_{3}(\lambda I-A)=\lambda^{3} I+\lambda^{2}\left[\begin{array}{llll}
0 & 1 & 1 & 0 \\
0 & 0 & 1 & 0 \\
0 & 0 & 0 & 1 \\
0 & 0 & 0 & 0
\end{array}\right]+\lambda\left[\begin{array}{llll}
0 & 0 & 1 & 1 \\
0 & 0 & 0 & 1 \\
0 & 0 & 0 & 0 \\
0 & 0 & 0 & 0
\end{array}\right]+\left[\begin{array}{llll}
0 & 0 & 0 & 1 \\
0 & 0 & 0 & 0 \\
0 & 0 & 0 & 0 \\
0 & 0 & 0 & 0
\end{array}\right] .
$$

Observe that

$$
p(\lambda)=\lambda^{3}+a \lambda^{2}+\frac{1}{2} \lambda \in W\left(C_{3}(\lambda I-A)\right)
$$

if and only if there exist $x=\left(x_{1}, x_{2}, x_{3}, x_{4}\right) \in \mathbf{C}^{4}$ such that

$$
\begin{aligned}
& x x^{*}=1 ; \\
& x_{1} \bar{x}_{4}=0 ; \\
& x_{1} \bar{x}_{3}+x_{1} \bar{x}_{4}+x_{2} \bar{x}_{4}=\frac{1}{2} ; \\
& x_{1} \bar{x}_{2}+x_{1} \bar{x}_{3}+x_{2} \bar{x}_{3}+x_{3} \bar{x}_{4}=a .
\end{aligned}
$$

By the second equation, $x_{1}=0$ or $x_{4}=0$. Substituting back to the first and third equations, we have either

(i) $x_{1} \bar{x}_{3}=\frac{1}{2}$ and $x_{2}=x_{4}=0$; or

(ii) $x_{2} \bar{x}_{4}=\frac{1}{2}$ and $x_{1}=x_{3}=0$.

It follows from the fourth equation that either $a=\frac{1}{2}$ or $a=0$. So

$$
p_{1}(\lambda)=\lambda^{3}+\frac{1}{2} \lambda^{2}+\frac{1}{2} \lambda, \quad p_{2}(\lambda)=\lambda^{3}+\frac{1}{2} \lambda \in W\left(C_{3}(\lambda I-A)\right)
$$

whereas $\left(p_{1}(\lambda)+p_{2}(\lambda)\right) / 2 \notin W\left(C_{3}(\lambda I-A)\right)$. Hence $W\left(C_{3}(\lambda I-A)\right)$ is not convex and cannot be equal to $P_{3}(\lambda I-A)$.

Concluding remarks. 1. Example 3 gives a negative answer to the question about the convexity of the set $W(\operatorname{adj}(\lambda I-A))$ raised by Johnson [3].

2. Observe that $C_{k}(\lambda I-A)$ is actually a matrix polynomial. Instead of looking at $W\left(C_{k}(\lambda I-A)\right)$, one may consider the numerical range of a matrix polynomial

$$
p(A)=\lambda^{m} A_{0}+\cdots+\lambda A_{m-1}+A_{m}
$$

Clearly, the set

$$
W(p(A))=\left\{x(p(A)) x^{*}: x \in \mathbf{C}^{n} \text { and } x x^{*}=1\right\}
$$

is essentially the joint range of the matrices $A_{0}, \ldots, A_{m}$, defined by

$$
\mathscr{W}\left(A_{0}, \ldots, A_{m}\right)=\left\{\left(x A_{0} x^{*}, \ldots, x A_{m} x^{*}\right): x \in \mathbf{C}^{n} \text { and } x x^{*}=1\right\} .
$$

As indicated in [1], the set $\mathscr{W}\left(A_{0}, \ldots, A_{m}\right)$ may fail to be convex even for hermitian matrices $A_{0}, \ldots, A_{m}$, if $m>2$.

3. Note that $C_{k}(A)$ is a particular type of induced matrix (see [4]). Our results may be generalized to other classes of induced matrices.

ACKNOWLEDgment. Thanks are due to Nam-Kiu Tsing for his helpful discussion. 


\section{REFERENCES}

1. Y. H. Au-Yeung and N. K. Tsing, An extension of the Hausdorff-Toeplitz theorem on the numerical range, Proc. Amer. Math. Soc. 89 (1983), 215-218.

2. K. Fan and G. Pall, Imbedding conditions for hermitian and normal matrices, Canad. J. Math. 9 (1957), 298-304.

3. C. R. Johnson, Interlacing polynomial, Proc. Amer. Math. Soc. 100 (1987), 401-404.

4. M. Marcus, Finite dimensional multilinear algebra. I and II, Marcel Dekker, 1973 and 1975.

5. M. Marcus and I. Filippenko, Linear operators preserving the decomposable numerical range, Linear and Multilinear Algebra 7 (1979), 27-36.

Department of Mathematics, University of Wisconsin, Madison, Wisconsin 53706

Current address: Department of Mathematics, College of William and Mary, Williamsburg, Virginia 23185 